\title{
Interplay of charge clustering and weak binding in reactions of ${ }^{8} \mathrm{Li}$
}

\author{
K. J. Cook,${ }^{1, *}$ I. P. Carter, ${ }^{1}$ E. C. Simpson, ${ }^{1}$ M. Dasgupta,,${ }^{1}$ D. J. Hinde,,${ }^{1}$ L. T. Bezzina,${ }^{1}$ Sunil Kalkal,${ }^{1,2}$ C. Sengupta,${ }^{1}$ \\ C. Simenel, ${ }^{1}$ B. M. A. Swinton-Bland, ${ }^{1}$ K. Vo-Phuoc, ${ }^{1}$ and E. Williams ${ }^{1}$ \\ ${ }^{1}$ Department of Nuclear Physics, Research School of Physics and Engineering, The Australian National University, Canberra, \\ Australian Capital Territory 2601, Australia \\ ${ }^{2}$ School of Physics and Material Sciences, Thapar University, Patiala-147004, India
}

(Received 25 October 2017; published 9 February 2018)

\begin{abstract}
In collisions of light, stable, weakly bound nuclides, complete fusion (capture of all of the projectile charge) has been found to be suppressed by $\sim 30 \%$ at above-barrier energies. This is thought to be related to their low thresholds for breakup into charged clusters. The observation of fusion suppression in the neutron-rich radioactive nucleus ${ }^{8} \mathrm{Li}$ is therefore puzzling: the lowest breakup threshold yields ${ }^{7} \mathrm{Li}+n$ which cannot contribute to fusion suppression because ${ }^{7} \mathrm{Li}$ retains all the projectile charge. In this work, the full characteristics of ${ }^{8} \mathrm{Li}$ breakup in reactions with ${ }^{209} \mathrm{Bi}$ are presented, including, for the first time, coincidence measurements of breakup into charged clusters. Correlations of cluster fragments show that most breakup occurs too slowly to significantly suppress fusion. However, a large cross section for unaccompanied $\alpha$ particles was found, suggesting that charge clustering, facilitating partial charge capture, rather than weak binding is the crucial factor in fusion suppression, which may therefore persist in exotic nuclides.
\end{abstract}

DOI: 10.1103/PhysRevC.97.021601

A stringent test of quantum many-body dynamics and tunneling is found in the fusion of atomic nuclei. Fusion involves the dissipation of kinetic energy of relative motion of the colliding nuclei into many internal degrees of freedom, ultimately producing an equilibrated compound nucleus. Despite the many-body nature of fusion, particular degrees of freedom, such as collective vibrations and rotations of the colliding nuclei, lead to orders-of-magnitude increase in tunneling probabilities [1,2]. In reactions of light nuclei (mass number $A \lesssim 10$ ), a different structure phenomenon becomes important - that of weak binding. The very strong binding energy of the $\alpha$ particle results in weakly bound ground-state cluster structures in nuclei such as ${ }^{9} \mathrm{Be}(\alpha+\alpha+n),{ }^{6} \mathrm{Li}$ $(\alpha+d)$ and ${ }^{7} \mathrm{Li}(\alpha+t)$. In contrast to the enhancement of fusion at below-barrier energies seen for heavy nuclei $[1,2]$, experiments have shown that complete fusion (experimentally defined [7] as capture of the complete charge of the projectile) with these weakly bound nuclei is suppressed by $\sim 30 \%$ at above-barrier energies [3-16].

The quantum dynamical origins of complete fusion suppression are not well understood. In the most weakly bound nuclei, the cluster projectiles readily break up, both via direct excitation above their breakup thresholds and through nucleon transfers to unbound states in neighboring nuclei $[17,18]$. Complete fusion may then be suppressed due to capture of only one cluster following charged-cluster breakup (incomplete fusion). Indeed, the presence of incomplete fusion products is often used as a proxy for complete fusion suppression [16].

The magnitude of complete fusion suppression has been shown in many cases to correlate with the threshold energy

*kaitlin.cook@anu.edu.au for direct breakup into two or more charged fragments [15]. However, recent investigations of breakup time scales suggest that only a small fraction of breakup occurs on a time scale short enough (i.e., sub-zeptosecond) such that breakup can occur prior to fusion, and so results in complete fusion suppression [19-21].

While the existence of fusion suppression is well established in reactions of stable weakly bound systems, complete fusion suppression in reactions of radioactive neutron-rich nuclei is not as well studied [4,22]. These nuclei have smaller neutron separation energies than the energy required for charged particle breakup. It is therefore expected that breakup dominantly takes the form of ${ }^{A} X \rightarrow^{A-x} X+x n$. Since complete fusion is operationally defined as capture of the complete charge of the projectile, breakup into one charged and one (or more) uncharged fragment(s) cannot contribute to incomplete charge capture. Thus, if breakup into two or more charged fragments prior to fusion is the mechanism for complete fusion suppression, then its magnitude should decrease as projectiles become more neutron rich. However, fusion of neutron-rich ${ }^{8} \mathrm{Li}$ [6] and ${ }^{10,11} \mathrm{Be}$ [23] has anomalously large suppression compared to the charged breakup thresholds. In particular, complete fusion of ${ }^{8} \mathrm{Li}$ in reactions with ${ }^{208} \mathrm{~Pb}$ is reported to show a $31 \%$ [6] suppression of complete fusion, though the charged breakup threshold is three times larger than ${ }^{6} \mathrm{Li}$ [5].

To understand the mechanism of fusion suppression in both stable and neutron-rich exotic nuclei, there is an urgent need to determine the relative importance of charged clustering and weak binding. This interplay can be investigated in reactions of ${ }^{8} \mathrm{Li}$. The breakup mode of ${ }^{8} \mathrm{Li}$ with the lowest threshold is ${ }^{8} \mathrm{Li} \rightarrow{ }^{7} \mathrm{Li}+n\left(S_{n}=2.03 \mathrm{MeV}\right)$. However, due to its proximity to strongly clustered nuclei such as ${ }^{7} \mathrm{Li}$, it may be that $\alpha$-cluster breakup following transfer is still significant. As 
${ }^{8} \mathrm{Li}$ is at the boundary between stable and neutron-rich exotic weakly bound nuclei, ${ }^{8} \mathrm{Li}$ provides an informative stepping stone toward more exotic systems.

In this paper, we present comprehensive measurements of charged particles for ${ }^{8} \mathrm{Li}+{ }^{209} \mathrm{Bi}$ at above-barrier energies to determine the breakup modes of ${ }^{8} \mathrm{Li}$, and what effect they can have on complete fusion. To achieve this, cross sections and characteristics of many direct reaction processes, as well as total reaction cross sections, have been determined.

The measurements used the solenoidal exotic rare isotope (SOLEROO) radioactive ion beam separator [24-27] at the Australian National University (ANU), based around a 6.5-T superconducting solenoid. Two position-sensitive parallel plate avalanche counters (PPACs) after the solenoid determine particle trajectories and masses, the latter through time of flight (TOF) both from the primary target and between the PPACs.

The secondary ${ }^{8} \mathrm{Li}$ beam was produced through the ${ }^{9} \mathrm{Be}\left({ }^{7} \mathrm{Li},{ }^{8} \mathrm{Li}\right){ }^{8} \mathrm{Be}$ reaction. The primary ${ }^{7} \mathrm{Li}$ beam of $45 \mathrm{MeV}$ and 50 pnA was pulsed, with 0.8 ns FWHM bunches being delivered every 107 ns by the ANU Heavy Ion Accelerator Facility's 14UD electrostatic accelerator. The primary beam impinged on a $5.57 \mathrm{mg} / \mathrm{cm}^{2}{ }^{9} \mathrm{Be}$ target, producing a ${ }^{8} \mathrm{Li}$ beam of $5 \times 10^{5}$ particles per second at the $1.25 \mathrm{mg} / \mathrm{cm}^{2}$ ${ }^{209} \mathrm{Bi}$ secondary target, angled at $45^{\circ}$ to the beam axis. ${ }^{8} \mathrm{Li}$ was transmitted in three energy groups, corresponding to population of its ground state $(57 \%)$, its first excited state at $0.98 \mathrm{MeV}$ [28] $(20 \%)$, and ${ }^{8} \mathrm{Be}$ in its first resonance at $3.03 \mathrm{MeV}$ [29] (23\%). After correction for energy loss, the secondary beam energy ranged between $E_{\mathrm{lab}}=38.24$ and $40.94 \mathrm{MeV}\left(1.27-1.36 E / V_{B}\right)$ at the center of the secondary target. Fusion is observed to be suppressed at these energies $[5,6]$, therefore they are appropriate for investigating the reactions associated with the suppression of complete fusion.

The relationship between the ${ }^{8} \mathrm{Li}$ ion trajectories through the solenoid (measured by the radial distance from the center of the downstream PPAC) and the ${ }^{8} \mathrm{Li}$ energies was established through a $\Delta E-E$ telescope that could be moved into the secondary target position. This relationship, shown in the inset to Fig. 1, enabled energy gating, allowing "one shot" measurements at several secondary beam energies. Further, since each ion trajectory was recorded, the incident angle and interaction point on the secondary target was used to reconstruct the true scattering angle on an event-by-event basis with $\pm 3^{\circ}$ resolution.

Particles were detected using two $\Delta E-E$ telescopes, with total solid angle $2.07 \mathrm{sr}$. Each consisted of a $400 \mu \mathrm{m}(\Delta E)$ and $500 \mu \mathrm{m}\left(E_{\text {residual }}\right) 60^{\circ}$ wedge-shaped double-sided silicon strip detector (DSSD) segmented into 16 arcs and 8 sectors, giving 256 pixels in both $\Delta E$ and $E_{\text {residual }}$. Each telescope extended from $\theta_{\text {lab }}=20.5^{\circ}$ to $90.3^{\circ}$, covering the largest angular range achievable around the grazing angle. Position, energy, and time were recorded for each event. Particles arising from reactions of ${ }^{8} \mathrm{Li}$ at the secondary target were separated from beam impurities by ensuring that their TOF was consistent with an origin from the secondary target (flight path $10 \mathrm{~cm}$ ) rather than the primary target (flight path $150 \mathrm{~cm}$ ).

Particles were identified by gating on TOF from the secondary target vs energy in the DSSD, and for $Z=1$ particles, gating also on $\Delta E-E$. Coincidence $\alpha+\alpha, \alpha+t, \alpha+d$, and

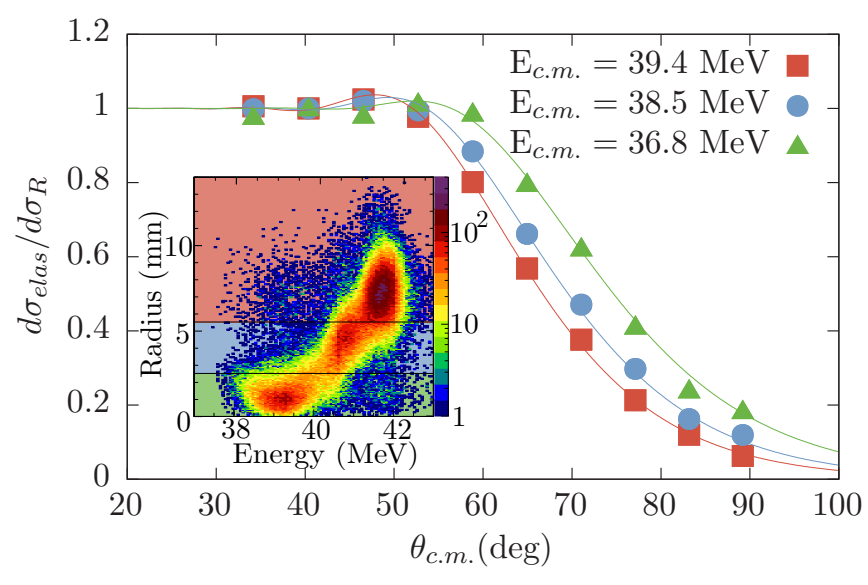

FIG. 1. Ratio of elastic to Rutherford scattering differential cross sections as a function of center-of-mass scattering angle $\theta_{\text {c.m. }}$. (main figure) for three different secondary beam energies extracted from the radial position of each event on the downstream PPAC (inset). The shading in the inset indicates the radial distances contributing to the curve of the same color in the main figure. The statistical error bars are smaller than the size of the points. The simultaneous optical model fit to all three data sets is shown by the lines.

$\alpha+p$ pairs were thus cleanly identified. For transfer reactions forming projectile-like nuclei in bound states, their characteristic energy-angle relationship allowed clean identification. $Z=1$ fragments were only identified if their energies were above the punch-through energy of the $\Delta E$ stage. These energy thresholds were accounted for in the efficiency correction for coincidence events (discussed below), giving complete coincidence cross sections.

We begin with an analysis of elastically scattered ${ }^{8} \mathrm{Li}$ to determine reaction cross sections. Normalization was through a Monte Carlo simulation of Rutherford scattering that incorporated the experimentally determined secondary beam spot size (9 $\mathrm{mm}$ FWHM) and divergence, as well as the angular coverage and resolution of the DSSDs.

The resulting elastic to Rutherford cross section ratios $d \sigma_{\text {elas }} / d \sigma_{R}$ are shown in Fig. 1 for the three secondary beam energies determined from the radial distance of events (Fig. 1 inset) from the center of the downstream PPAC. Optical model fits were performed to extract reaction cross sections. To reduce the number of free parameters, the real and imaginary radii were fixed at $r_{0}=1.25 \mathrm{fm}$ and $r_{w}=1.35 \mathrm{fm}$, with $a_{0}=$ $0.65 \mathrm{fm}$ and $a_{w}=0.75 \mathrm{fm}$. The real and imaginary potential depths were adjusted to simultaneously fit data at the three energies. The resulting reaction cross sections are 1257, 1414, and $1491 \mathrm{mb}$ at $E_{\text {c.m. }}=36.8,38.5$, and $39.4 \mathrm{MeV}$ respectively, and $1423 \mathrm{mb}$ at the average center-of-mass energy of $38.6 \mathrm{MeV}$. As a result of simultaneous fitting, the statistical uncertainty on each is $\pm 9 \mathrm{mb}$, with an estimated systematic uncertainty of $\pm 20 \mathrm{mb}$. With a calculated total fusion cross section [30] at the average beam energy of $1045 \mathrm{mb}$, this leaves $\sim 380 \mathrm{mb}$ for direct reactions.

The simplest of these are nucleon transfer reactions forming bound states of ${ }^{7} \mathrm{Li}$ and ${ }^{9} \mathrm{Be}$. Differential cross sections are shown (beam energy averaged) in Fig. 2(c). The yields peak 


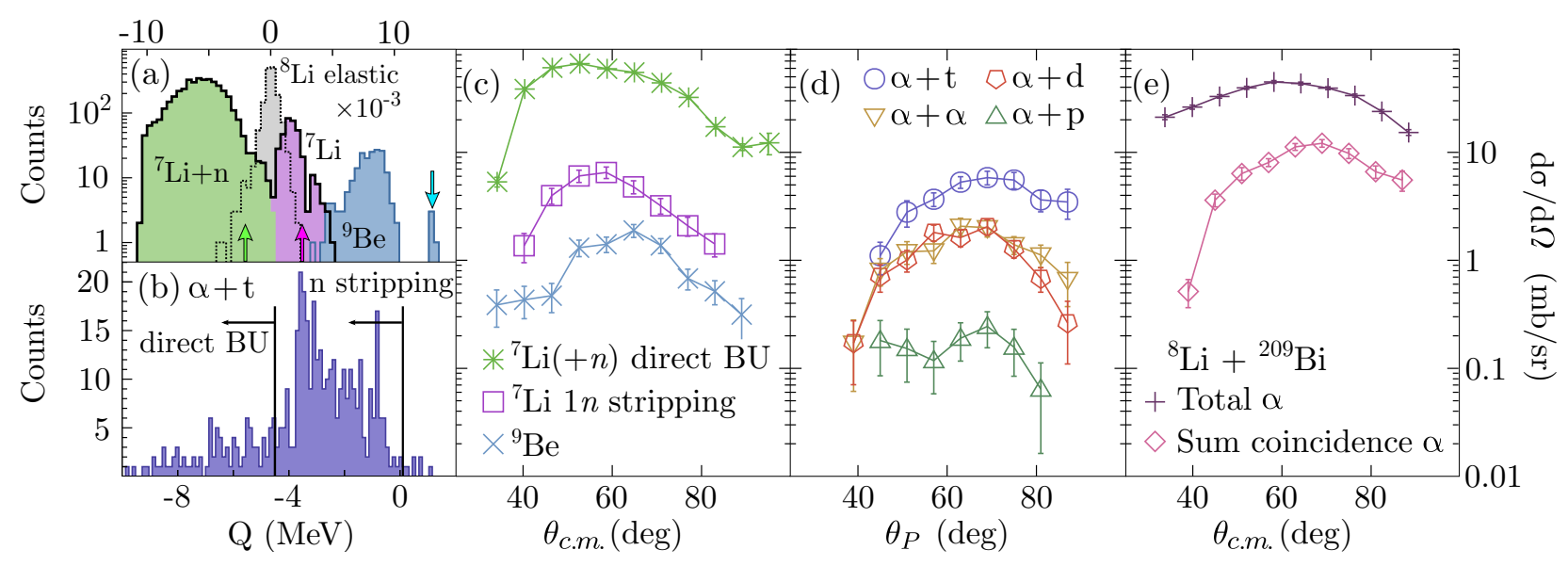

FIG. 2. $Q$-value spectra for (a) singles events and (b) $\alpha+t$ pairs measured for ${ }^{8} \mathrm{Li}+{ }^{209} \mathrm{Bi}$. The thresholds for each process are indicated by arrows of the same color. From these yields, energy-averaged differential cross sections for singles (c) and coincidence (d) events were determined. The total $\alpha$ production cross section is compared to that of $\alpha$ produced in coincidence with another charged fragment (e). The error bars represent the statistical uncertainty in the experimental data, along with a small contribution from the simulation. All peak around the grazing angle (Fig. 1). Lines guide the eye.

near the grazing angle. Integrating over the measured angular range, the ${ }^{9} \mathrm{Be}$ cross section was $5.0 \pm 0.6 \mathrm{mb}$. From the angular distributions for this and other transfer and breakup channels, the contribution outside the experimental angular range is estimated to be $\sim 10 \%$.

For ${ }^{7} \mathrm{Li}$, the measured $Q$-value spectrum [thick line in Fig. 2(a)] allowed differentiation between formation by neutron stripping (purple) ( $Q$-value of $+2.57 \mathrm{MeV})$ and direct breakup (green) $(Q=-2.03 \mathrm{MeV})$. Integrating the angular distributions [Fig. 2(c)] gives a cross section for ${ }^{7} \mathrm{Li}$ production via neutron stripping of $16.1 \pm 1.7 \mathrm{mb}$. The direct breakup (BU) cross section of $219 \pm 5 \mathrm{mb}$ is the largest contributor to the direct reaction cross section.

Reactions forming two charged fragments are those that may result in incomplete fusion. Coincidence yields of $\alpha+$ $\alpha, p, d, t$ were efficiency corrected as described in Ref. [19] and cross sections were extracted normalizing to Rutherford scattering. The resulting differential cross sections are shown in Fig. 2(d) as a function of the reconstructed center-of-mass angle of the unbroken projectile-like nucleus $\theta_{P}$ [19]. These represent the cross sections for transfer to an unbound state where neither fragment is captured. $\alpha+t$ production is dominant $(\sigma=19 \pm 2 \mathrm{mb}$ ), with the $\alpha+d, \alpha+\alpha$, and $\alpha+p$ cross sections being $\sigma=6.2 \pm 0.9,5.6 \pm 0.7$, and $\sigma=0.9 \pm 0.4$ $\mathrm{mb}$, respectively.

Breakup into two charged fragments can lead to complete fusion suppression if breakup occurs before the projectile-like nucleus passes inside the fusion barrier. Thus, suppression of complete fusion requires breakup time scales of $\lesssim$ zeptoseconds. Recent works [19-21] have shown that the energy and angular correlations of breakup fragments are sensitive to these time scales, and that inclusion of the lifetimes of resonant states in model calculations is essential to reproduce experimental results for subbarrier breakup.

For ${ }^{8} \mathrm{Li}+{ }^{209} \mathrm{Bi}, \alpha+t$ breakup is dominant and hence we restrict the detailed discussion of time scales to $\alpha+t$ fragments. They can be produced either by neutron stripping, forming ${ }^{7} \mathrm{Li}$ in an unbound state, followed by its subsequent breakup $(Q=0.1 \mathrm{MeV})$, or by direct excitation of $\alpha+t+n$ continuum states $(Q=-4.5 \mathrm{MeV})$ where the neutron also carries away some energy. The reconstructed $Q$-value [31] spectrum [Fig. 2(b)] shows that $78 \pm 6 \%$ of events have $Q$ values more positive than those for direct breakup $(Q>$ $-4.5 \mathrm{MeV})$. The remainder may arise either through direct breakup or from neutron stripping resulting in a target-like excitation energy greater than $4.6 \mathrm{MeV}$ (which is seen for ${ }^{9} \mathrm{Be}$ $+{ }^{209} \mathrm{Bi}$ neutron stripping reactions [19]).

The relative energy $E_{\text {rel }}$ between breakup fragments reflects the excitation energy and lifetime of the projectile-like nucleus [21,31]. The measured $\alpha+t$ relative energy spectrum is presented in Fig. 3(a) (red points), showing a well-defined peak at $2.19 \mathrm{MeV}$. Events in this peak correspond to breakup of ${ }^{7} \mathrm{Li}$ from its $7 / 2^{-}$state $\left[E_{x}=4.652 \mathrm{MeV} \tau=\hbar / \Gamma\left(E_{x}\right)=\right.$ $\left.10^{-20} \mathrm{~s}\right][20,29]$. The vertical black line indicates the expected position of the peak in $E_{\text {rel }}$ from this state. The small number of events extending to large $E_{\text {rel }}$ can be associated with breakup that occurs close to the target-like nucleus [21,31]. They comprise a small fraction of the total. In contrast, for measurements of direct ${ }^{7} \mathrm{Li}$ breakup after interactions with ${ }^{209} \mathrm{Bi}$, detailed kinematic modeling demonstrated that the overwhelming majority of breakup occurred close to the target-like nucleus [20]. This shows that the particular nuclear reaction, as well as the identity of the intermediate nucleus, is important in determining time scales of breakup.

A quantitative estimate of the fraction of breakup producing $\alpha+t$ pairs prior to the distance of closest approach is obtained by performing a classical dynamical simulation for this breakup mode. This approach is justified since classical trajectory models including resonant lifetimes successfully reproduce below-barrier energy and angular correlations of breakup fragments that are sensitive to the location (and thus time) of breakup [19-21]. Our simulation includes a realistic description of the excitation energy and lifetime distribution of the projectile-like nucleus, and detector resolution effects [20]. 

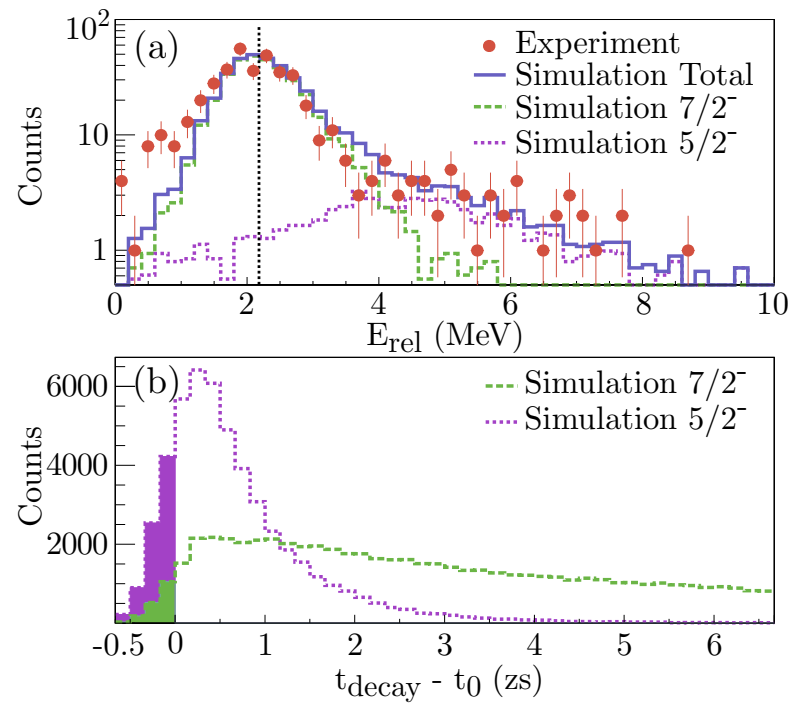

FIG. 3. (a) Measured $E_{\text {rel }}$ distribution (red points) and classical dynamical simulation (blue line) for $\alpha+t$ pairs produced after interactions of ${ }^{8} \mathrm{Li}$ with ${ }^{209} \mathrm{Bi}$. The vertical dashed line indicates the expected location of the peak in $E_{\text {rel }}$ from the first $7 / 2^{-}$resonance of ${ }^{7} \mathrm{Li}$. The simulation includes contributions from the $7 / 2^{-}$(green) and $5 / 2^{-}$(purple) state of ${ }^{7} \mathrm{Li}$. From this simulation, the breakup time relative to the time of closest approach is shown in (b). The breakup that occurs prior to the time of closest approach is shaded.

Incorporating $65 \pm 6 \%$ of strength in the $7 / 2^{-}$resonance of ${ }^{7} \mathrm{Li}$ (green line) and $35 \pm 6 \%$ in the $5 / 2^{-}$resonance (purple line, $E_{x}=6.604 \mathrm{MeV}, \tau=7 \times 10^{-22} \mathrm{~s}$ [29]), gives a good fit to the experimental distribution. The total $E_{\text {rel }}$ distribution, shown by the blue line in Fig. 3(a) reproduces the data satisfactorily. The discrepancy at low $E_{\text {rel }}$ represents a small fraction of the distribution and may be alleviated by including contributions from the nonresonant continuum [20]. However, it was not pursued further as low $E_{\text {rel }}$ events arise mainly from slow breakup.

From this simulation, the time of breakup $t_{\text {decay }}$ relative to the time $t_{0}$ at the distance of closest approach $\left(t_{\text {decay }}-t_{0}\right)$ can be extracted, and is shown in Fig. 3(b). For these events on grazing trajectories, only $7 \pm 1 \%$ of breakup occurs prior to the distance of closest approach (shaded region). Given that this fraction should not change significantly as trajectories become more central, it follows that reactions producing $\alpha+t$ cannot significantly suppress complete fusion.

The cross sections for reactions forming $\alpha+\alpha$ and $\alpha+d$ are a factor of $\sim 3$ smaller than those forming $\alpha+t$ pairs. In both cases, a substantial amount of breakup is through long-lived resonances of ${ }^{8} \mathrm{Be}$ and ${ }^{6} \mathrm{Li}$, respectively. Therefore, the capacity of breakup forming $\alpha+\alpha$ and $\alpha+d$ pairs to suppress complete fusion through incomplete charge capture is also limited. While reactions forming $\alpha+p$ pairs go through ${ }^{5} \mathrm{Li}$, which has a very short lifetime, the total cross section of $0.9 \pm 0.4 \mathrm{mb}$ precludes any significant contribution to incomplete fusion. It is possible that $\alpha+p$ pairs may also be produced from direct breakup to ${ }^{7} \mathrm{He}+p$ decaying to $\alpha+$ $2 n+p$; however, a significant contribution from this process is not supported by the reconstructed $Q$-value spectrum.

The deduced differential cross section for production of $\alpha$ from all breakup reactions is shown in Fig. 2(e) (pink diamonds). Those for singles $\alpha$ particles are shown by purple crosses. Integrating over the experimental angular coverage, the $37 \pm 2 \mathrm{mb}$ cross section of $\alpha$ from charged cluster coincidences only explains a small part of the total $\alpha$ cross section of $198 \pm 6 \mathrm{mb}$. The remaining $161 \pm 6 \mathrm{mb}$ must be due to $\alpha$ particles that are not accompanied by another charged fragment. This large cross section of unaccompanied $\alpha$ particles, also seen in Ref. [32], is associated with the production of polonium isotopes. These products had generally been believed to be formed following breakup and incomplete charge capture. The long time scales of breakup determined in this work require an alternative mechanism to explain the large unaccompanied $\alpha$ yields and the observed fusion suppression [6]. Unaccompanied $Z=1$ particles (not measurable in this work) would result in the production of astatine isotopes, contributing further to the yield of incomplete charge capture products.

In conclusion, the measurements for ${ }^{8} \mathrm{Li}+{ }^{209} \mathrm{Bi}$ presented here demonstrate for the first time that ${ }^{8} \mathrm{Li}$ breakup into charged clusters is both diverse and significant. Cross sections of no-capture breakup forming two charged fragments were measured, namely, $\alpha+t, \alpha+\alpha, \alpha+d$, and $\alpha+p$. Remarkably, ${ }^{8} \mathrm{Li}$ shows as much diversity in breakup modes as was observed with ${ }^{6,7} \mathrm{Li}$ [31], demonstrating the continued significance of charged clustering in reaction outcomes, even when the neutron separation energy is low.

Measured particle-particle correlations of breakup fragments show breakup occurs on time scales sufficiently long that breakup prior to reaching the distance of closest approach is insignificant. This has an important consequence: it appears that the observed $31 \%$ suppression of complete fusion [6] cannot be explained by reduction of the number of intact projectiles due to breakup into charged fragments before reaching the capture barrier.

What mechanism, then, gives rise to the large yield of unaccompanied $\alpha$ particles? It may be that clustering in the ground state of ${ }^{8} \mathrm{Li}[33$ ] leads to one charged fragment interacting more strongly with the target than the other. Partial charge transfer to the target, facilitated by this interaction, without the need for breakup, may then be a key mechanism leading to fusion suppression. This needs further further investigation, since as long as charged cluster structure persists in neutron-rich radioactive nuclei [34-41], suppression of complete fusion [23] may also be expected.

This work was supported by the Australian Research Council grants FL110100098, DP170102423, FT120160760, DE140100784, DP130101569 and DP160101254. Support for ANU Heavy Ion Accelerator Facility operations through the NCRIS program is acknowledged.
[1] C. H. Dasso, S. Landowne, and A. Winther, Nucl. Phys. A 405, 381 (1983).
[2] J. R. Leigh, M. Dasgupta, D. J. Hinde, J. C. Mein, C. R. Morton, R. C. Lemmon, J. P. Lestone, J. O. Newton, 
H. Timmers, J. X. Wei, and N. Rowley, Phys. Rev. C 52, 3151 (1995).

[3] M. Dasgupta, D. J. Hinde, S. L. Sheehy, and B. Bouriquet, Phys. Rev. C 81, 024608 (2010).

[4] L. Canto, P. R. S. Gomes, R. Donangelo, J. Lubian, and M. Hussein, Phys. Rep. 596, 1 (2015).

[5] M. Dasgupta, P. R. S. Gomes, D. J. Hinde, S. B. Moraes, R. M. Anjos, A. C. Berriman, R. D. Butt, N. Carlin, J. Lubian, C. R. Morton, J. O. Newton, and A. Szanto de Toledo, Phys. Rev. C 70, 024606 (2004).

[6] E. F. Aguilera, E. Martinez-Quiroz, P. Rosales, J. J. Kolata, P. A. DeYoung, G. F. Peaslee, P. Mears, C. Guess, F. D. Becchetti, J. H. Lupton, and Y. Chen, Phys. Rev. C 80, 044605 (2009).

[7] M. Dasgupta, D. J. Hinde, R. D. Butt, R. M. Anjos, A. C. Berriman, N. Carlin, P. R. S. Gomes, C. R. Morton, J. O. Newton, A. Szanto de Toledo, and K. Hagino, Phys. Rev. Lett. 82, 1395 (1999).

[8] C. Signorini, T. Glodariu, Z. H. Liu, M. Mazzocco, M. Ruan, and F. Soramel, Prog. Theor. Phys. Suppl. 154, 272 (2004).

[9] P. K. Rath, S. Santra, N. L. Singh, R. Tripathi, V. V. Parkar, B. K. Nayak, K. Mahata, R. Palit, S. Kumar, S. Mukherjee, S. Appannababu, and R. K. Choudhury, Phys. Rev. C 79, 051601 (2009).

[10] C. Signorini, Z. Liu, Z. Li, K. Löbner, L. Müller, M. Ruan, K. Rudolph, F. Soramel, C. Zotti, A. Andrighetto, L. Stroe, A. Vitturi, and H. Zhang, Eur. Phys. J. A 5, 7 (1999).

[11] P. R. S. Gomes, I. Padron, E. Crema, O. A. Capurro, J. O. Fernández Niello, A. Arazi, G. V. Martí, J. Lubian, M. Trotta, A. J. Pacheco, J. E. Testoni, M. D. Rodríguez, M. E. Ortega, L. C. Chamon, R. M. Anjos, R. Veiga, M. Dasgupta, D. J. Hinde, and K. Hagino, Phys. Rev. C 73, 064606 (2006).

[12] C. S. Palshetkar, S. Santra, A. Chatterjee, K. Ramachandran, S. Thakur, S. K. Pandit, K. Mahata, A. Shrivastava, V. V. Parkar, and V. Nanal, Phys. Rev. C 82, 044608 (2010).

[13] Y. D. Fang, P. R. S. Gomes, J. Lubian, M. L. Liu, X. H. Zhou, D. R. Mendes Junior, N. T. Zhang, Y. H. Zhang, G. S. Li, J. G. Wang, S. Guo, Y. H. Qiang, B. S. Gao, Y. Zheng, X. G. Lei, and Z. G. Wang, Phys. Rev. C 91, 014608 (2015).

[14] A. Mukherjee, Subinit Roy, M. Pradhan, M. Saha Sarkar, P. Basu, B. Dasmahapatra, T. Bhattacharya, S. Bhattacharya, S. Basu, A. Chatterjee, V. Tripathi, and S. Kailas, Phys. Lett. B 636, 91 (2006).

[15] L. R. Gasques, D. J. Hinde, M. Dasgupta, A. Mukherjee, and R. G. Thomas, Phys. Rev. C 79, 034605 (2009).

[16] N. T. Zhang, Y. D. Fang, P. R. S. Gomes, J. Lubian, M. L. Liu, X. H. Zhou, G. S. Li, J. G. Wang, S. Guo, Y. H. Qiang, Y. H. Zhang, D. R. Mendes Junior, Y. Zheng, X. G. Lei, B. S. Gao, Z. G. Wang, K. L. Wang, and X. F. He, Phys. Rev. C 90, 024621 (2014).

[17] D. H. Luong, M. Dasgupta, D. J. Hinde, R. du Rietz, R. Rafiei, C. J. Lin, M. Evers, and A. Diaz-Torres, Phys. Rev. C 88, 034609 (2013).

[18] R. Rafiei, R. du Rietz, D. H. Luong, D. J. Hinde, M. Dasgupta, M. Evers, and A. Diaz-Torres, Phys. Rev. C 81, 024601 (2010).

[19] K. J. Cook, E. C. Simpson, D. H. Luong, S. Kalkal, M. Dasgupta, and D. J. Hinde, Phys. Rev. C 93, 064604 (2016).

[20] S. Kalkal, E. C. Simpson, D. H. Luong, K. J. Cook, M. Dasgupta, D. J. Hinde, I. P. Carter, D. Y. Jeung, G. Mohanto, C. S.
Palshetkar, E. Prasad, D. C. Rafferty, C. Simenel, K. Vo-Phuoc, E. Williams, L. R. Gasques, P. R. S. Gomes, and R. Linares, Phys. Rev. C 93, 044605 (2016).

[21] E. C. Simpson, K. J. Cook, D. H. Luong, S. Kalkal, I. P. Carter, M. Dasgupta, D. J. Hinde, and E. Williams, Phys. Rev. C 93, 024605 (2016).

[22] B. B. Back, H. Esbensen, C. L. Jiang, and K. E. Rehm, Rev. Mod. Phys. 86, 317 (2014).

[23] D. J. Hinde and M. Dasgupta, Phys. Rev. C 81, 064611 (2010).

[24] R. Rafiei, D. J. Hinde, M. Dasgupta, D. C. Weisser, A. G. Muirhead, A. B. Harding, A. K. Cooper, H. J. Wallace, N. R. Lobanov, A. Wakhle, M. L. Brown, C. J. Lin, A. J. Horsley, R. Du Rietz, D. H. Luong, and M. Evers, Nucl. Instrum. Methods Phys. Res., Sect. A 631, 12 (2011).

[25] A. Horsley, D. J. Hinde, M. Dasgupta, R. Rafiei, A. Wakhle, M. Evers, D. H. Luong, and R. du Rietz, Nucl. Instrum. Methods Phys. Res., Sect. A 646, 174 (2011).

[26] I. P. Carter, K. Ramachandran, M. Dasgupta, D. J. Hinde, R. Rafiei, D. H. Luong, E. Williams, K. J. Cook, S. McNeil, D. C. Rafferty, A. B. Harding, A. G. Muirhead, and T. Tunningley, EPJ Web Conf. 63, 02022 (2013).

[27] I. P. Carter, M. Dasgupta, D. J. Hinde, D. H. Luong, E. Williams, K. Ramachandran, K. J. Cook, A. G. Muirhead, S. Marshall, and T. Tunningley, EPJ Web Conf. 91, 00001 (2015).

[28] D. R. Tilley, J. H. Kelley, J. L. Godwin, D. J. Millener, J. E. Purcell, C. G. Sheu, and H. R. Weller, Nucl. Phys. A 745, 155 (2004).

[29] D. Tilley, C. Cheves, J. Godwin, G. Hale, H. Hofmann, J. Kelley, C. Sheu, and H. Weller, Nucl. Phys. A 708, 3 (2002).

[30] L. C. Chamon, B. V. Carlson, L. R. Gasques, D. Pereira, C. De Conti, M. A. G. Alvarez, M. S. Hussein, M. A. Cândido Ribeiro, E. S. Rossi, and C. P. Silva, Phys. Rev. C 66, 014610 (2002).

[31] D. H. Luong, M. Dasgupta, D. J. Hinde, R. Du Rietz, R. Rafiei, C. J. Lin, M. Evers, and A. Diaz-torres, Phys. Lett. B 695, 105 (2011).

[32] J. J. Kolata, V. Z. Goldberg, L. O. Lamm, M. G. Marino, C. J. O’Keeffe, G. Rogachev, E. F. Aguilera, H. García-Martínez, E. Martinez-Quiroz, P. Rosales, F. D. Becchetti, T. W. O’Donnell, D. A. Roberts, J. A. Brown, P. A. DeYoung, J. D. Hinnefeld, and S. A. Shaheen, Phys. Rev. C 65, 054616 (2002).

[33] W. Nörtershäuser, R. Sánchez, G. Ewald, A. Dax, J. Behr, P. Bricault, B. A. Bushaw, J. Dilling, M. Dombsky, G. W. F. Drake, S. Götte, H. J. Kluge, T. Kühl, J. Lassen, C. D. P. Levy, K. Pachucki, M. Pearson, M. Puchalski, A. Wojtaszek, Z.-C. Yan, and C. Zimmermann, Phys. Rev. A 83, 012516 (2011).

[34] Y. Kanada-En'yo, Phys. Rev. C 94, 024326 (2016).

[35] M. Ito and K. Ikeda, Rep. Prog. Phys. 77, 096301 (2014).

[36] F. Kobayashi and Y. Kanada-En'yo, Phys. Rev. C 89, 024315 (2014)

[37] Y. Kanada-En'yo, M. Kimura, and A. Ono, Prog. Theor. Exp. Phys. 2012, 01A202 (2012).

[38] W. von Oertzen, M. Freer, and Y. Kanada-En'yo, Phys. Rep. 432, 43 (2006).

[39] P. Descouvemont, Nucl. Phys. A 699, 463 (2002).

[40] Y. Kanada-En'yo and H. Horiuchi, Prog. Theor. Phys. Suppl. 142, 205 (2001).

[41] M. Seya, M. Kohno, and S. Nagata, Prog. Theor. Phys. 65, 204 (1981). 